\title{
INFLUENCE OF CONVERTER CAPACITANCE IN RESONANCE- BASED SENSORLESS SWITCHED RELUCTANCE DRIVES
}

\author{
K.R. Geldhof, A. Van den Bossche and J.A.A. Melkebeek \\ Department of Electrical Energy, Systems and Automation (EESA) \\ Ghent University (UGent), Sint-Pietersnieuwstraat 41, B-9000 Gent, Belgium \\ phone: +32 (0)9 264 3442, fax: +32 (0)9 2643582 \\ e-mail: Kristof.Geldhof@UGent.be
}

Keywords: Switched Reluctance Motor, Position Estimation, Resonance, Test Pulse, Parasitic Capacitance

\begin{abstract}
By observation of electrical resonances, switched reluctance drives can be controlled without position sensor. The range of resonance frequencies is defined by the position-dependent phase inductance and a parasitic capacitance, mainly associated with the converter switches. In this paper, an analytical model is used to predict the aligned resonance frequency, taking into account material and geometry factors, as well as dynamic effects such as eddy current losses. The range of resonance frequencies can be influenced by mounting external capacitors over the power semiconductor devices. It is shown that an optimal aligned frequency is associated with a trade-off between a high position resolution and a high pulse-width modulation frequency. Good correspondence is obtained between the model-predicted results and measurements on a switched reluctance drive.
\end{abstract}

\section{Introduction}

Position- or speed-sensorless control of switched reluctance machines receives a lot of interest from the industrial and academic community.

One class of sensorless methods for switched reluctance drives is based on resonance between the inductance of an idle motor phase and an external capacitor [6]. The resonance frequency depends on the phase inductance, which varies with the rotor position. If a high-frequency sinusoidal current is injected in the resonant circuit, the amplitude and phase of a voltage in the circuit is modulated by the rotor position.

A rotor position estimation method based on electrical resonance, but without the need for an external excitation circuit, is proposed in [4]. The method is characterized by the fact that very short voltage pulses are used to trigger resonance between the magnetic energy stored in the phase inductances and the energy stored in parasitic capacitances associated with the power semiconductor devices, power cables and motor phase windings.

This paper presents an analytical model for the resonant circuit associated with an aligned rotor position. Parasitic capacitances, motor geometry, material parameters and eddy current losses in the magnetic laminations are taken into account to predict the aligned resonance frequency of the drive. It is shown that this frequency should be minimized to obtain a maximum position resolution between the unaligned and aligned rotor position. However, due to increasing measurement delay with decreasing frequencies, the aligned resonance frequency is bounded by the time between switching actions of the power semiconductor devices, and thus by the PWM period.

The range of resonance frequencies can be influenced by mounting external capacitors in parallel with the power semiconductor devices. A simple setup is proposed to measure the different capacitances in the system. Measurements show the validity of the proposed model.

\section{Position Estimation in switched reluctance drives}

In switched reluctance drives, the rotor position can be obtained by observation of the unforced oscillation of a resonant circuit comprising the inductance and parasitic capacitance associated with an idle motor phase [4]. Fig. 1(a) shows an asymmetric $\mathrm{H}$-bridge of a switched reluctance drive converter and a motor phase. If the IGBTs and freewheeling diodes are blocked, these devices behave as a parasitic capacitance. The model of Fig. 1(b) shows these parasitic capacitances, indicated by $C_{\mathrm{i}}$ and $C_{\mathrm{d}}$ respectively, and the parasitic capacitance $C_{\mathrm{w}}$ of the motor phase winding and the capacitance $C_{\mathrm{c}}$ associated with the power cable between converter and motor phase. The impedance $Z_{\mathrm{w}}$ represents the phase winding impedance without the contribution of the parasitic capacitance $C_{\mathrm{w}}$.

The circuit of Fig. 1(b) can be excited by application of a short voltage pulse, see Fig. 2(a). During the short switch-on of the IGBTs, the parasitic capacitances $C_{\mathrm{d}}, C_{\mathrm{c}}$ and $C_{\mathrm{w}}$ are charged up to the bus bar voltage $V_{\mathrm{dc}}$. At the end of the voltage pulse, a resonance is initiated due to an exchange of energy between the parasitic capacitances and the phase inductance $L_{\mathrm{w}}$. This resonance is damped, mainly due to eddy currents in the magnetic material. Fig. 2(b) shows the position-dependence of the resonance. If the rotor is in the aligned position with respect to the idle phase, the phase inductance has a maximum value and the corresponding (undamped) resonance frequency will reach a minimum value, given by

$$
\omega_{\mathrm{res}}=\frac{1}{\sqrt{L_{\mathrm{w}}\left(\theta, \omega_{\mathrm{res}}\right) C_{\mathrm{eq}}}},
$$




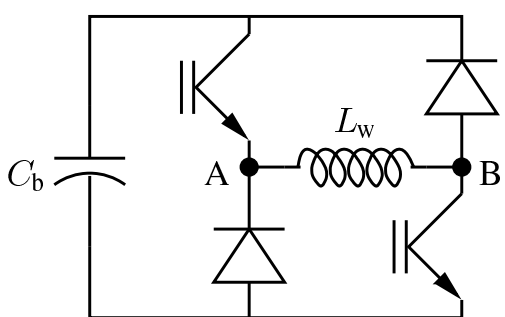

(a)

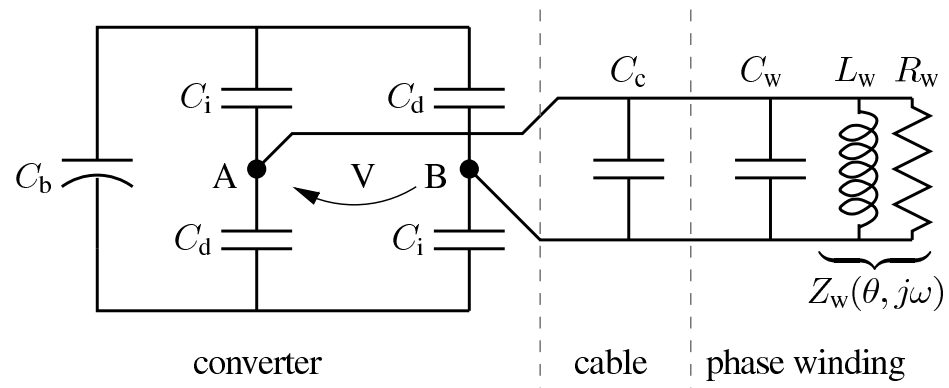

(b)

Figure 1. Converter H-bridge with phase winding (a) and parasitic model of motor-converter combination (b).

where $C_{\mathrm{eq}}$ represents the total equivalent parasitic capacitance:

$$
C_{\mathrm{eq}}=\frac{C_{\mathrm{i}}+C_{\mathrm{d}}}{2}+C_{\mathrm{c}}+C_{\mathrm{w}} .
$$

It can be seen from Fig. 2 that the timing of the test pulse is chosen in such a way that the first part of the resonance waveform is not disturbed by the switching actions in the active (torque-producing) phase. From this part of the waveform, rotor position can be estimated e.g. by measuring the damped oscillation period, or by a single voltage measurement at a fixed time after the start of the test pulse [4].

\section{Analytical Resonance Frequency Model}

\subsection{Aligned Resonance Frequency}

If the frequency-dependency of the phase inductance would be neglected in (1), the resonance frequency would be inversely proportional to $\sqrt{L_{\mathrm{w}}(\theta)}$. The ratio of unaligned to aligned resonance frequency would therefore be independent of the equivalent capacitance $C_{\text {eq }}$ :

$$
\frac{\omega_{\text {res,un }}}{\omega_{\text {res,al }}}=\sqrt{\frac{L_{\mathrm{a}}}{L_{\mathrm{u}}}},
$$

with $L_{\mathrm{a}}$ and $L_{\mathrm{u}}$ the (frequency-independent) aligned and unaligned phase inductance respectively. However, in a sinusoidal regime at frequency $\omega$, the phase inductance depends on $\omega$ due to induced eddy currents in the magnetic laminations of the motor. If a winding with $w$ turns is wound around a core which consists of $n$ adjacent rectangular-shaped magnetic sheets, the core impedance $Z_{\mathrm{c}}(j \omega)$ is defined by [1] [7]

$$
Z_{\mathrm{c}}(j \omega)=2 n \frac{b}{l} w^{2} Z_{0}(j \omega) \tanh \left(\gamma(j \omega) \frac{d}{2}\right),
$$

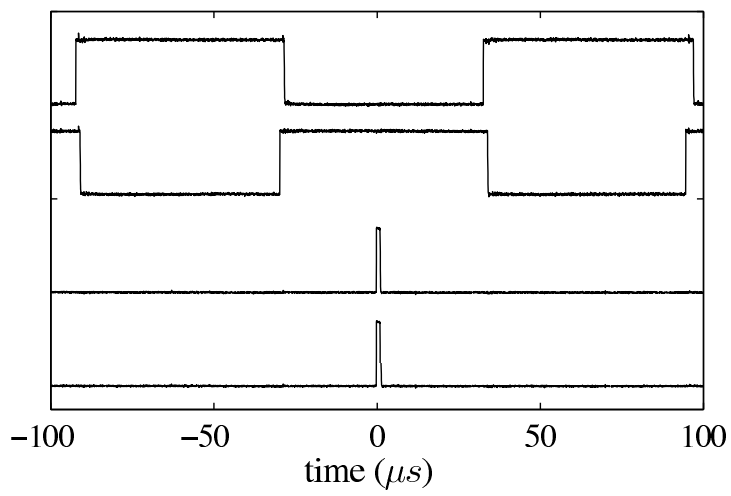

(a) Control signals for IGBTs of phase A (top) and IGBTs of phase C (bottom).

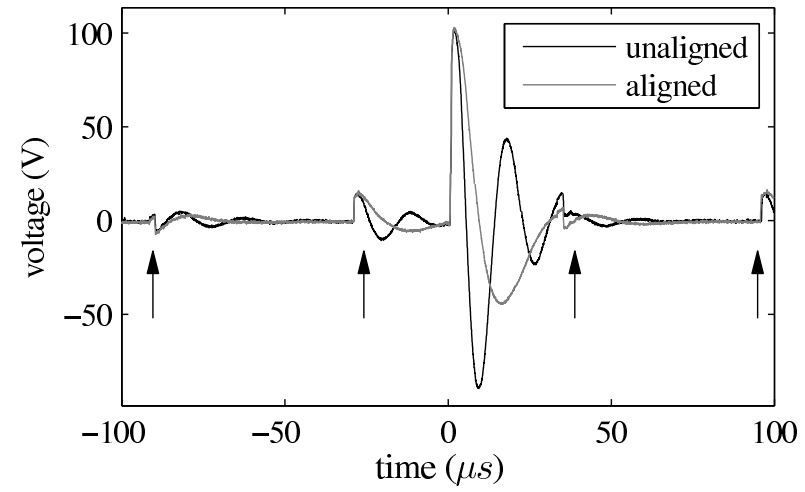

(b) Voltage resonance in phase $\mathrm{C}$. The arrows indicate distortions due to switching actions in phase A.

Figure 2. Application of $1.2 \mu \mathrm{s}$ voltage pulse in phase $\mathrm{C}$, for the unaligned and aligned position. Phase A is current-controlled. $V_{\mathrm{dc}}=105 \mathrm{~V}$.

where $j$ is the imaginary unit and $b, l$ and $d$ represent the width, length and thickness of a sheet respectively. The characteristic impedance $Z_{0}(j \omega)$ of the magnetic material and the propagation function $\gamma(j \omega)$ are defined by

$$
\begin{array}{r}
Z_{0}(j \omega)=\left(\frac{j \omega \mu}{\sigma}\right)^{1 / 2}, \\
\gamma(j \omega)=(j \omega \mu \sigma)^{1 / 2},
\end{array}
$$

with $\mu$ and $\sigma$ the magnetic permeability and electrical conductivity of the material respectively.

For low frequencies, the equality

$$
\lim _{z \rightarrow 0} \frac{\tanh (z)}{z}=1
$$

can be used to reduce (4) to its low-frequency limit $Z_{\mathrm{CLF}_{\mathrm{LF}}}$ :

$$
\lim _{\omega \rightarrow 0} Z_{\mathrm{c}}=Z_{\mathrm{c}_{\mathrm{LF}}}=j \omega \cdot \mu w^{2} \frac{b n d}{l},
$$

which corresponds to a constant low-frequency inductance $L_{\mathrm{c}_{\mathrm{LF}}}$ :

$$
L_{\mathrm{c}_{\mathrm{LF}}}=\mu w^{2} \frac{b n d}{l} \text {. }
$$

In order to model the impedance of the magnetic material in a switched reluctance motor, (9) can be used, provided that $l$ and 


\begin{tabular}{l|c|r|l} 
core and geometry data & symbol & value & unit \\
\hline relative permeability & $\mu_{r}$ & 2000 & - \\
sheet conductivity & $\sigma$ & $3 \cdot 10^{6}$ & $\mathrm{~S} / \mathrm{m}$ \\
sheet thickness & $d$ & 0.5 & $\mathrm{~mm}$ \\
number of sheets & $n$ & 205 & - \\
number of turns & $w$ & 208 & - \\
average magnetic path length & $l$ & 300 & $\mathrm{~mm}$ \\
average magnetic path width & $b$ & 17.55 & $\mathrm{~mm}$ \\
stator stack length & $l_{s}$ & 100.8 & $\mathrm{~mm}$ \\
stator yoke width & $b_{y}$ & 13 & $\mathrm{~mm}$ \\
stator tooth width & $b_{p}$ & 20 & $\mathrm{~mm}$ \\
single air gap length & $l_{a}$ & 0.4 & $\mathrm{~mm}$ \\
unaligned inductance & $L_{\mathrm{u}}$ & 10 & $\mathrm{mH}$ \\
natural system capacitance & $C_{\mathrm{eq}}$ & 490 & $\mathrm{pF}$
\end{tabular}

Table I

MAGNETIC CORE, GEOMETRY AND CONVERTER DATA

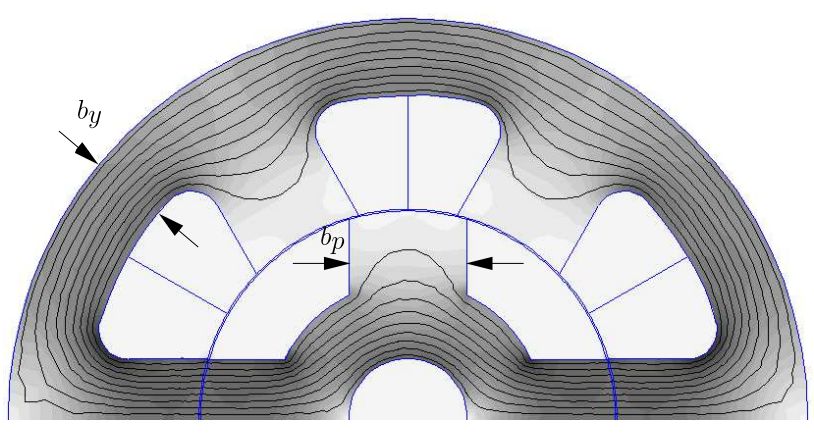

Figure 3. Switched reluctance motor geometry.

$b$ correspond to the average length and width of the magnetic path in the core of the machine [3]. This is especially true in the aligned position, where fringing effects can be neglected.

In an aligned position, the magnetic path includes a double air gap, the inductance of which is given by

$$
L_{\text {air }}=\frac{\mu_{0} w^{2} l_{s} b_{p}}{2 l_{\text {air }}},
$$

with $l_{\text {air }}$ the single air gap length, $b_{p}$ the stator pole width as indicated in Fig. 3, and $l_{s}$ the length of the stator lamination stack $\left(l_{s}=n d\right.$ if the isolation distance between the magnetic laminations of the core is neglected).

In the case that the aligned motor phase is idle, the corresponding equivalent circuit is shown in Fig. 4. The low-frequency aligned phase inductance is therefore determined by the parallel circuit of the inductances (9) and (10).

In the case of frequencies $\omega$ exceeding the cut-off frequency of the material, following equality holds:

$$
\omega \gg \frac{4}{\mu \sigma d^{2}} \Longleftrightarrow \tanh \left(\gamma(j \omega) \frac{d}{2}\right) \rightarrow 1
$$

From the motor data of Table I, it follows that the magnetic material has a cut-off frequency of $338 \mathrm{~Hz}$. The aligned resonance waveform of Fig. 2(b) has a frequency of $32.9 \mathrm{kHz}$. For most motor-converter combinations it can be assumed that (11) holds, resulting in a simplified expression for the core impedance $Z_{\mathrm{c}}$ :

$$
Z_{\mathrm{c}}(j \omega)=2 n \frac{b}{l} w^{2} \frac{1+j}{\sqrt{2}} \sqrt{\frac{\mu}{\sigma}} \sqrt{\omega} .
$$

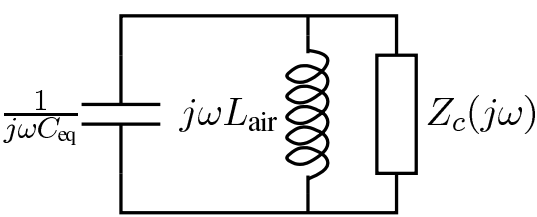

Figure 4. Equivalent circuit for idle motor phase connected to converter bridge.

The impedance (12) has a real part, which represents the eddycurrent induced damping in the magnetic core. In the following this damping will be neglected, so that the resonance frequency can be calculated by means of (1), valid for the undamped system. In this case, the core impedance (12) reduces to its imaginary part

$$
Z_{\mathrm{c}}(j \omega)=j \omega L_{c}(\omega)=j \sqrt{2} n \frac{b}{l} w^{2} \sqrt{\frac{\mu}{\sigma}} \sqrt{\omega} .
$$

By defining the constant $K_{\mathrm{c}_{\mathrm{HF}}}$,

$$
K_{\mathrm{c}_{\mathrm{HF}}}=\sqrt{2} n \frac{b}{l} w^{2} \sqrt{\frac{\mu}{\sigma}}
$$

the core inductance can be written as

$$
L_{c}(\omega)=\frac{K_{\mathrm{c}_{\mathrm{HF}}}}{\sqrt{\omega}}
$$

As can be seen from Fig. 4, the parallel circuit of (10) and (15) yields the phase inductance $L_{\mathrm{w}}$ :

$$
L_{\mathrm{w}}(\omega)=\frac{L_{c}(\omega) L_{\mathrm{air}}}{L_{c}(\omega)+L_{\mathrm{air}}} .
$$

Substitution of (16) in (1) results in a non-linear equation, the solution of which is the undamped aligned resonance frequency:

$$
\frac{1}{\omega^{2}}=\frac{K_{\mathrm{c}_{\mathrm{HF}}} L_{\mathrm{air}}}{K_{\mathrm{cHF}}+L_{\mathrm{air}} \sqrt{\omega}} C_{\mathrm{eq}} .
$$

This equation can be rewritten as

$$
K_{\mathrm{cHF}} C_{\mathrm{eq}} \omega^{2}-\sqrt{\omega}=\frac{K_{\mathrm{c}_{\mathrm{HF}}}}{L_{\mathrm{air}}} .
$$

If $\sqrt{\omega} \gg K_{\mathrm{c}_{\mathrm{HF}}} / L_{\mathrm{air}}$ the high-frequency solution of (18) can be obtained:

$$
\omega_{\text {res,al }} \rightarrow\left(\frac{1}{K_{\mathrm{c}_{\mathrm{HF}}} C_{\mathrm{eq}}}\right)^{2 / 3} .
$$

With the parameters of Table I, the right-hand side of (18) equals 156 , while (19) yields $33.4 \mathrm{kHz}$. The high-frequency assumption and equation (19) are therefore valid for the motor-converter combination under test, even in the case that $C_{\mathrm{eq}}$ would be much larger than the value specified in Table I.

\subsection{Unaligned Resonance Frequency}

The unaligned rotor position is characterized by a large air gap which is the dominating reluctance in the magnetic path. Therefore, the unaligned phase inductance $L_{\mathrm{u}}$ remains almost constant over a wide frequency range, resulting in an unaligned resonance frequency $\omega_{\text {res, un }}$ which is inversely proportional to $\sqrt{L_{\mathrm{u}}}$ :

$$
\omega_{\text {res,un }}=\frac{1}{\sqrt{L_{\mathrm{u}} C_{\mathrm{eq}}}} .
$$




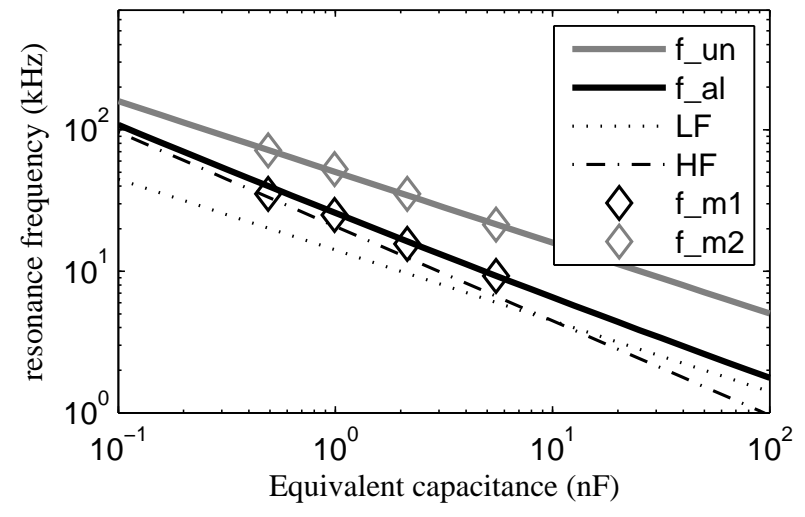

Figure 5. Calculated unaligned resonance frequency (f_un), aligned resonance frequency (f_al), low-frequency (LF) and high-frequency (HF) approximation of aligned resonance frequency as a function of equivalent capacitance, measurements of unaligned (f_m1) and aligned (f_m2) resonance frequency.

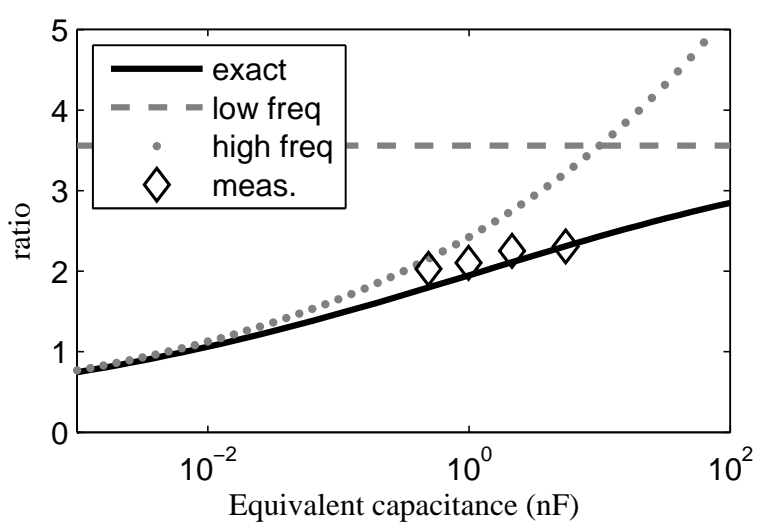

Figure 6. Calculated ratio of unaligned to aligned resonance frequency: exact solution according to (18), calculated low-frequency (22) and high-frequency approximation (21), and measured ratio.

\subsection{Ratio of Resonance Frequencies}

The unaligned and aligned resonance frequency as a function of the equivalent capacitance $C_{\mathrm{eq}}$ are shown in Fig. 5. The figure also shows how the aligned resonance frequency curve converges towards its high-frequency approximation (for low values of $C_{\mathrm{eq}}$ ) and towards its low-frequency approximation (for high values of $C_{\text {eq }}$ ). It is clear that at low frequencies, the unaligned and aligned curves run parallel in the logarithmic diagram, and the ratio of unaligned to aligned resonance frequency remains at the constant value (3). For decreasing capacitance values, the aligned resonance frequency rises faster than the unaligned resonance frequency, leading to curves which converge towards higher frequencies. Theoretically, the curves intersect near the point $(5 \mathrm{pF}, 700 \mathrm{kHz})$. However, a capacitance as low as $20 \mathrm{pF}$ is not to occur in a converter-machine combination in the power range of hundreds of Watts or more. Furthermore, a frequency of hundreds of $\mathrm{kHz}$ is likely to be above the eigenfrequency of a machine phase winding in the considered power range. Above this eigenfrequency, the winding behaves more capacitive than inductive, and the model (4) is not valid anymore.

\section{Trade-off of Capacitance Value}

The ratio of unaligned to aligned resonance frequency is shown in Fig. 6. The high-frequency behaviour of the ratio follows from (19) and (20):

$$
\frac{\omega_{\text {res, unaligned }}}{\omega_{\text {res,aligned }}}=\frac{K_{\mathrm{c}_{\mathrm{HF}}}^{2 / 3}}{\sqrt{L_{\mathrm{u}}}} C^{1 / 6} .
$$

For high capacitance values the ratio converges to the lowfrequency approximation:

$$
\frac{\omega_{\text {res,unaligned }}}{\omega_{\text {res,aligned }}}=\sqrt{\frac{\frac{L_{\mathrm{air}} L_{\mathrm{LLF}}}{L_{\text {air }}+L_{\mathrm{cF}}}}{L_{u}}} .
$$

As discussed in 3.1, the high-frequency approximation (21) is valid for practically all motor-converter combinations.

Both from Fig. 5 and (21) it can be concluded that the equivalent capacitance should be taken as large as possible, in order to maximize the ratio of the unaligned to aligned resonance frequency, and thus to maximize the position resolution between the unaligned and aligned rotor position. However, a trade-off has to be made with regard to the PWM period at which the machine is controlled. From Fig. 2(b) it can be seen that the voltage resonance waveform gets distorted at time instants where switching actions take place in the active (current-controlled) phase of the machine. However, the waveform should not be distorted during the time which is necessary to extract position information from the waveform. For a given modulation strategy, this puts a limit on the maximum PWM frequency that can be used, as the time between successive switching actions decreases linearly with the PWM period. In a worst-case scenario, a large amount of capacitance is present in the system, and the PWM frequency has to be reduced so much that significant current ripple and/or audible noise cannot be avoided anymore.

\section{Model Validation}

\subsection{System Capacitance Measurement}

For the calculation of the analytical model, the capacitance $C_{\mathrm{eq}}$ has to be known. Following measurements are made to retrieve the values of the capacitances in (2):

- The combined cable-winding capacitance is measured by means of an LRC meter: $C_{\mathrm{c}}+C_{\mathrm{w}}=390 \mathrm{pF}$.

- The capacitance of an IGBT and a diode is measured by means of the setup of Fig. 7. A bias voltage between 0 and $200 \mathrm{~V}$ can be applied over the device under test (D.U.T.) by means of a dc source. A function generator imposes a $10 \mathrm{kHz}, 1 \mathrm{~V}$ peak-to-peak square wave voltage $V_{\text {gen }}$ over the D.U.T. An oscilloscope probe (with capacitance $C_{\mathrm{sc}}$ ) is used to measure the voltage $V_{\mathrm{m}}$ over a known capacitor $C_{\mathrm{t}}$. A $100 \mathrm{nF}$ capacitor decouples the bias voltage from the scope probe. The waveforms $V_{\text {gen }}$ and $V_{\mathrm{m}}$ are shown in Fig. 8. The step changes in $V_{\mathrm{m}}$ are determined by the capacitive division between the output capacitance of the D.U.T. and the combined test and probe capacitance:

$$
\frac{V_{\mathrm{m}}}{V_{\text {gen }}} \approx \frac{C_{\text {D.U.T. }}}{C_{\text {D.U.T. }}+\left(C_{\mathrm{t}}+C_{\mathrm{sc}}\right)}
$$


The test capacitance $C_{\mathrm{t}}$ is chosen in such a way that the denominator (23) is approximately twice the output capacitance of the D.U.T., resulting in a good measurement resolution. The exponential behaviour between the step changes is determined by the time constant of the combined test/probe capacitance and the high-impedance resistor with value $100 \mathrm{k} \Omega$.

From (23) the output capacitance $C_{\text {D.U.T. }}$ can be calculated as a function of the bias voltage over the component. Fairchild smart power modules of the type FCSA50SN60 were used in the converter under test. The resulting $(C, V)$ profile of an IGBT in a module is shown in Fig. 9. The profile has a highly non-linear dependence on the bias voltage over the component. The physical origin lies in the depletion layer, the thickness of which varies strongly as a function of the bias voltage [2].

For the analytical model it is assumed that a large part of the resonance takes place in a time interval in which the voltage over the phase winding is relatively small compared to the initial voltage $V_{\mathrm{dc}}$. After the resonance has damped out, the phase voltage becomes zero, and the voltage over the power semiconductor devices is $V_{\mathrm{dc}} / 2$. From Fig. 9 it can be seen that the $(C, V)$ profile is quite flat in the region around $V_{\mathrm{dc}} / 2=100 \mathrm{~V}$. Therefore, the value of $C_{\text {D.U.T. }}$ at $V_{\mathrm{dc}} / 2$ yields a good approximation for the output capacitance of the device.

For the converter under test, capacitance values of $160 \mathrm{pF}$ and $40 \mathrm{pF}$ were measured for an IGBT and diode respectively. The equivalent converter capacitance $C_{\mathrm{eq}}$ is therefore given by $\left(C_{\mathrm{i}}+C_{\mathrm{d}}\right) / 2=100 \mathrm{pF}$.

The total system capacitance (2) consists of the capacitances of the converter, motor cable and phase winding. With the mentioned values a total capacitance $C_{\mathrm{eq}}=490 \mathrm{pF}$ is obtained.

\subsection{Increasing System Capacitance}

For the motor under test, the equivalent capacitance of the system is well below the threshold where a conflict arises between the aligned resonance frequency and the PWM period. This means that there is enough reserve to increase $C_{\text {eq }}$ in order to obtain an increased position resolution. A possible way to achieve a higher equivalent capacitance is by mounting external ( $\mathrm{d} v / \mathrm{d} t$-type) capacitors $C_{\text {ext }}$ in parallel with the active semiconductor devices, as shown in Fig. 10. In this way, the equivalent capacitance (2) is increased by $C_{\text {ext }} / 2$ :

$$
C_{\mathrm{eq}}=\frac{\left(C_{\mathrm{i}}+C_{\mathrm{ext}}\right)+C_{\mathrm{d}}}{2}+C_{\mathrm{c}}+C_{\mathrm{w}} .
$$

Disadvantages of the external capacitors are the extra cost and extra space required on the printed circuit board. Advantages include:

- Higher position resolution.

- Increased linearity of the system. If the external capacitance dominates the non-linear capacitance $C_{\mathrm{i}}+C_{\mathrm{d}}$, the equivalent capacitance (24) will be highly voltage-independent. This could be important in series production, where the system capacitance could be maintained within certain tolerances.

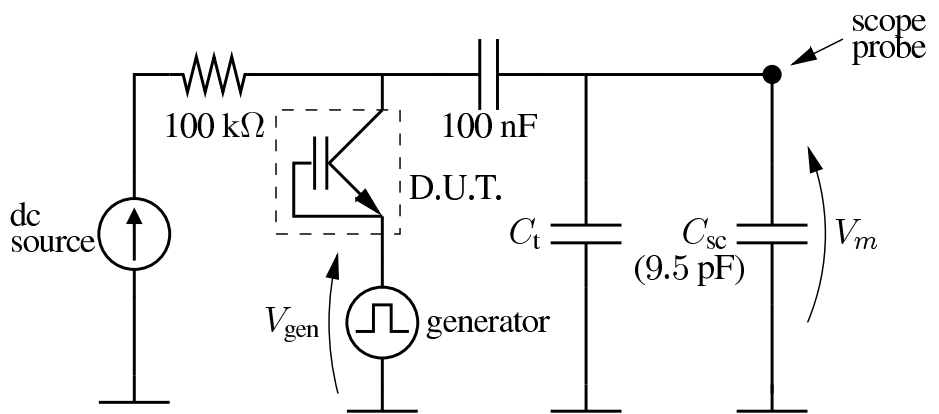

Figure 7. IGBT output capacitance measurement setup.

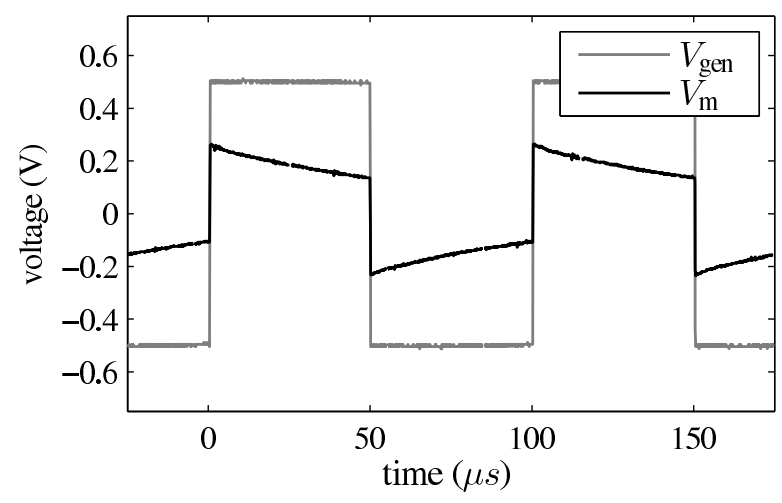

Figure 8. Measured waveforms in IGBT output capacitance measurement setup.

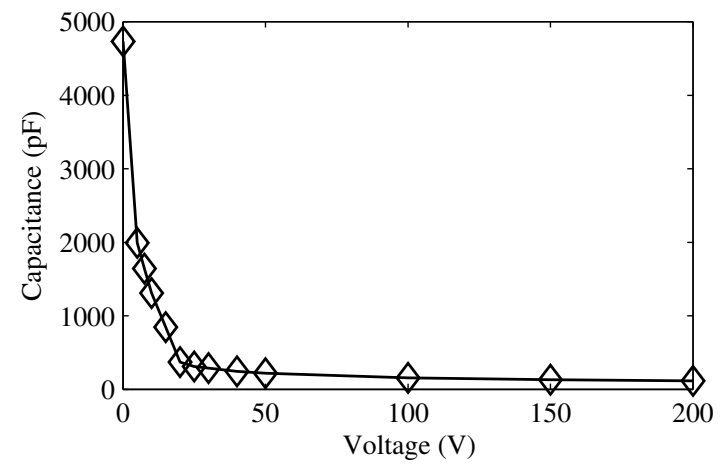

Figure 9. Measured IGBT output capacitance vs collector-emittor voltage.

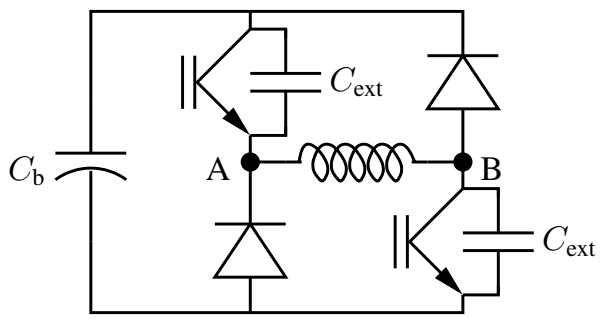

Figure 10. Converter H-bridge with external capacitors placed in parallel with IGBTs. 


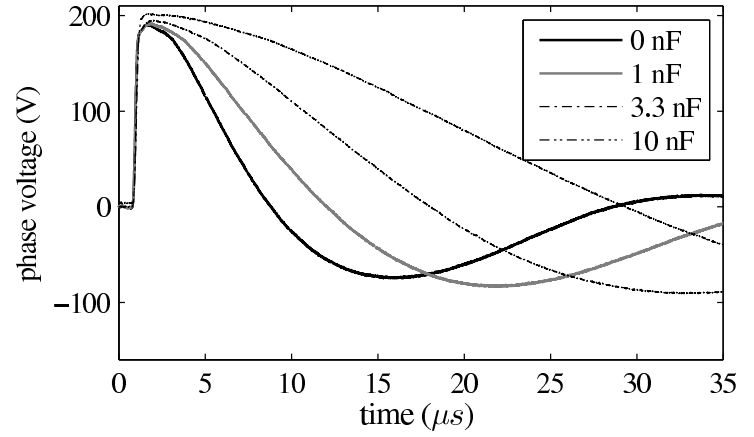

(a) aligned

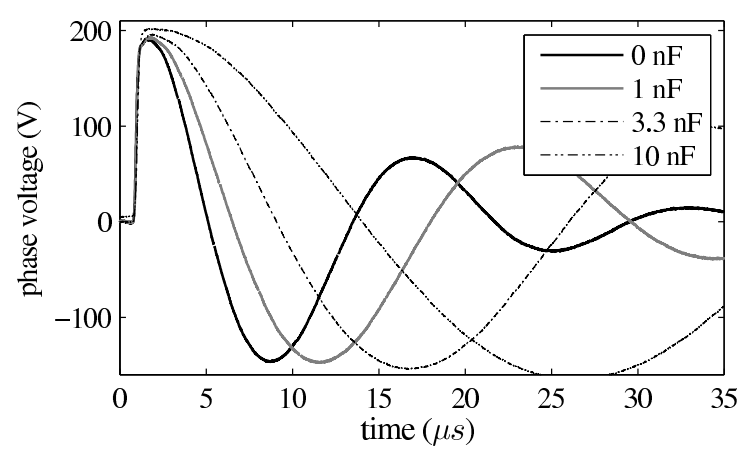

(b) unaligned

Figure 11. Aligned (a) and unaligned (b) phase voltage waveforms in response to $1 \mu \mathrm{s}$ testpulses for different external capacitance values $C_{\mathrm{ext}} . V_{\mathrm{dc}}=200 \mathrm{~V}$.

- Decreased switching losses in the IGBTs. The slight increase in switch-on loss, associated with the energy loss $1 / 2 C_{\mathrm{ext}}\left(V_{\mathrm{dc}} / 2\right)^{2}$ is overcompensated by the decrease in switch-off loss [5].

- Reduced common-mode currents and associated electromagnetic interference due to reduced $\mathrm{d} v / \mathrm{d} t$ over the power semiconductor devices.

In order to validate the model for the aligned resonance frequency, three capacitor pairs with respective values $1 \mathrm{nF}, 3.3 \mathrm{nF}$ and $10 \mathrm{nF}$ are placed in parallel with the IGBTs of the converter, as shown in Fig. 10. Test pulses with a duration of $1 \mu \mathrm{s}$ are applied to an idle motor phase and the subsequent phase voltage resonances are measured, see Fig. 11. The first zero crossing of these (damped) waveforms is assumed to be at $1 / 4$ th of the resonance period. From Fig. 11(a), the aligned resonance frequencies are measured and they are listed in Table II. These data are also visualized with markers in Figs. 5 and 6. A good correspondence is obtained between the measured curves $\left(C_{\text {eq }}, f_{\text {res,aligned }}\right)$ and the predicted curves from the analytical model (18),(20), even though the damping is neglected in the analytical model. From the measured data in Fig. 6 it can be seen that the ratio of unaligned to aligned resonance frequency increases with increasing capacitance values, which stresses the point that this ratio is not constant due to induced eddy currents in the magnetic laminations.

\begin{tabular}{r|l}
$C_{\mathrm{eq}}$ & $f_{\text {res,aligned }}$ \\
\hline $0.49 \mathrm{nF}+0=0.49 \mathrm{nF}$ & $35.2 \mathrm{kHz}$ \\
$0.49 \mathrm{nF}+1 / 2 \mathrm{nF}=0.99 \mathrm{nF}$ & $25.0 \mathrm{kHz}$ \\
$0.49 \mathrm{nF}+3.3 / 2 \mathrm{nF}=2.14 \mathrm{nF}$ & $15.6 \mathrm{kHz}$ \\
$0.49 \mathrm{nF}+10 / 2 \mathrm{nF}=5.49 \mathrm{nF}$ & $9.3 \mathrm{kHz}$
\end{tabular}

Table II

MEASURED ALIGNED RESONANCE FREQUENCY AS A FUNCTION OF TOTAL SYSTEM CAPACITANCE

\section{Conclusion}

An analytical model is presented which allows to predict the range of resonance frequencies in a resonance-based sensorless SRM drive. For a known geometry, converter and material data, the model can be used to tune the total system capacitance in order to make a trade-off between a high position resolution and a high PWM frequency. The tuning of this capacitance can be realized by mounting external capacitors in parallel with the parasitic capacitance of the power semiconductor devices. Experiments with different system capacitance values were performed. A good correspondence is achieved between the measured resonance frequencies and the predicted values with the analytical model.

\section{References}

[1] G. Bertotti. Hysteresis in Magnetism. Academic Press, San Diego, CA, 1998.

[2] S. Dimitrijev. Understanding Semiconductor Devices. Oxford University Press, New York, N.Y., 2000.

[3] K. R. Geldhof, A. Van den Bossche and J. A. A. Melkebeek. "Influence of eddy currents on resonance-based position estimation of switched reluctance drives", International Conference on Electrical Machines and Systems, ICEMS 2008, pages 2820-2825, Wuhan, China, Oct. 17-20, 2008.

[4] K. R. Geldhof, A. Van den Bossche and J. A. A. Melkebeek. "Rotor position estimation of switched reluctance motors based on damped voltage resonance", IEEE Trans. Ind. Electron., 57(3), mar 2010.

[5] P. Haaf and J. Harper. Understanding diode reverse recovery and its effect on switching losses. Fairchild On-Demand Webinar, http://www.techonline.com/learning/webinar/202802635.

[6] P. Laurent, M. Gabsi and B. Multon. "Sensorless rotor position analysis using resonant method for switched reluctance motor", Conference Record of the 1993 IEEE Industry Applications Society Annual Meeting, volume 1, pages 687694, Toronto, Canada, Oct. 1993.

[7] A. Van den Bossche and V. C. Valchev. Inductors and Transformers for Power Electronics. CRC-press, Boca Raton, USA, 2005. 